OPEN ACCESS

Check for updates

\title{
Effect of reductions in amyloid levels on cognitive change in randomized trials: instrumental variable meta-analysis
}

\author{
Sarah F Ackley, ${ }^{1}$ Scott C Zimmerman, ${ }^{1}$ Willa D Brenowitz, ${ }^{1,2}$ Eric J Tchetgen Tchetgen, ${ }^{3}$ \\ Audra L Gold, ${ }^{1}$ Jennifer J Manly, ${ }^{4}$ Elizabeth Rose Mayeda, ${ }^{5}$ Teresa J Filshtein, ${ }^{6}$ Melinda C Power, ${ }^{7}$ \\ Fanny M Elahi, ${ }^{8}$ Adam M Brickman, ${ }^{4}$ M Maria Glymour ${ }^{1}$
}

${ }^{1}$ Department of Epidemiology and Biostatistics, University of California, San Francisco, 550 16th Street, San Francisco, CA USA

${ }^{2}$ Department of Psychiatry, University of California, San Francisco, CA, USA

${ }^{3}$ Department of Statistics, University of Pennsylvania, PA, USA

${ }^{4}$ Taub Institute for Research on Alzheimer's Disease and the Aging Brain, G H Sergievsky

Center, Department of

Neurology, Columbia University,

New York, NY, USA

${ }^{5}$ Department of Epidemiology,

University of California, Los

Angeles, CA, USA

${ }^{6} 23 \&$ Me, Sunnyvale, CA, USA

${ }^{7}$ Department of Epidemiology,

George Washington University,

Milken Institute School of Public

Health, Washington DC, USA

${ }^{8}$ UCSF Weill Institute for

Neurosciences, University of California, San Francisco, CA, USA

Correspondence to: M M Glymour maria.slymour@ucsf.edu (or @MariaGlymour on Twitter; ORCID 0000-0001-9644-3081)

Additional material is published online only. To view please visit the journal online.

Cite this as: $B M J$ 2021;372:n156 http://dx.doi.org/10.1136/bmj.n156

Accepted: 21 December 2020

\section{ABSTRACT}

OBJECTIVE

To evaluate trials of drugs that target amyloid to determine whether reductions in amyloid levels are likely to improve cognition.

DESIGN

Instrumental variable meta-analysis.

SETTING

14 randomized controlled trials of drugs for the prevention or treatment of Alzheimer's disease that targeted an amyloid mechanism, identified from ClinicalTrials.gov.

POPULATION

Adults enrolled in randomized controlled trials of amyloid targeting drugs. Inclusion criteria for trials vary, but typically include adults aged 50 years or older with a diagnosis of mild cognitive impairment or Alzheimer's disease, and amyloid positivity at baseline.

\section{MAIN OUTCOME MEASURES}

Analyses included trials for which information could be obtained on both change in brain amyloid levels measured with amyloid positron emission tomography and change in at least one cognitive test score reported for each randomization arm.

RESULTS

Pooled results from the 14 randomized controlled trials were more precise than estimates from any single trial. The pooled estimate for the effect of reducing amyloid levels by 0.1 standardized uptake value ratio units was an improvement in the mini-mental state examination score of 0.03 ( $95 \%$ confidence interval -0.06 to 0.1 ) points. This study provides a web application that allows for the re-estimation of the results when new data become available and illustrates the magnitude of the new evidence that would be necessary to achieve a pooled

\section{WHAT IS ALREADY KNOWN ON THIS TOPIC}

Most trials of drugs targeting amyloid for the treatment of Alzheimer's disease have not shown statistically significant cognitive benefit

Individual trials do not, however, provide conclusive evidence about the potential impact of amyloid reduction on cognitive decline

Evidence from multiple trials of amyloid targets has not been systematically combined

\section{WHAT THIS STUDY ADDS}

Pooled estimates from 14 randomized controlled trials of drugs to reduce amyloid levels suggest no substantial improvement in cognition within the timeframe of typical clinical trials estimate supporting the benefit of reducing amyloid levels.

\section{CONCLUSIONS}

Pooled evidence from available trials reporting both reduction in amyloid levels and change in cognition suggests that amyloid reduction strategies do not substantially improve cognition.

\section{Introduction}

Amyloid plaques and oligomers are hypothesized to cause a cascade of pathological events resulting in cognitive decline in Alzheimer's disease. ${ }^{1-3}$ Motivated by the amyloid cascade hypothesis, a primary aim of many new treatments for the prevention or management of Alzheimer's disease has been to reduce amyloid $\beta$ levels in the brain. ${ }^{4}$ Although the presence of amyloid plaques and oligomers in the brain is highly correlated with the progression of Alzheimer's disease, ${ }^{5}{ }^{6}$ the mechanisms by which amyloid might mediate neuronal pathology are currently not well understood. ${ }^{7}$ To date, no anti-amyloid treatments have progressed sufficiently to receive approval from the Food and Drug Administration (the regulatory agency for pharmaceuticals in the United States). ${ }^{8}$ Drugs have targeted various amyloid species-amyloid plaques, amyloid oligomers, and soluble oligomers-and have been performed in populations with mild to moderate Alzheimer's disease, as well as earlier stages of disease (prodromal Alzheimer's disease). ${ }^{9}$ Most trials targeting amyloid failed to produce positive results in either early or late stages of the disease. The negative findings from these trials have prompted skepticism about amyloid's role in neuronal disease, and many have instead argued that amyloid could be a marker for other disease processes and therefore is not a viable drug target. ${ }^{1011}$

No single trial has, however, provided conclusive evidence about the potential impact of reduction in amyloid levels on cognitive decline. Most trials have been powered to evaluate specific treatments and were not designed to accurately estimate the effect of amyloid reduction itself on cognitive outcomes. Individual trials generally have been small, and often data on amyloid burden has been assessed only in a subsample of participants (eg, trials reviewed $\mathrm{in}^{12}$ ). For any individual trial of a drug targeting amyloid that fails to deliver cognitive benefits, several alternative explanations exist: amyloid reduction might not improve cognition, or the mechanism by which amyloid is targeted might affect whether and the extent to which reductions in brain amyloid levels slow cognitive decline $\left(\mathrm{eg},{ }^{13}\right)$. Alternatively, 
the benefit of amyloid reduction might be too small to detect within an individual trial, a plausible situation because the expense of trials often limits the number of participants. ${ }^{9}$ This lack of statistical power (ie, the chance an effect of any given size would lead to a statistically significant result in the study) to detect an effect could be overcome by combining results from multiple trials of different drugs that all target amyloid. For example, findings from any one study might have wide confidence intervals that would not allow the ruling out of either substantial benefit or substantial harm. Combining evidence from multiple trials provides more precise effect estimates (narrower confidence intervals). To date, evidence from multiple trials of amyloid targets has yet to be systematically combined, and trial data have not been leveraged to evaluate whether changes in amyloid levels are likely to improve cognition. Using a modification of an intention-to-treat meta-analysis based on instrumental variable analyses, ${ }^{14}$ the results of multiple studies can be aggregated into one combined estimate of the effect of decreasing amyloid levels on cognitive decline.

We analyzed individually and in aggregate changes in brain amyloid levels and changes in cognition using data from randomized trials of amyloid reducing drug treatments to estimate the effect of reductions in amyloid levels on change in cognition. Using randomization as an instrumental variable for amyloid reduction, we evaluated the plausibility of the hypothesis that reductions in amyloid levels slow cognitive decline. We also aggregated results across multiple studies of drugs and evaluated the plausibility of differential effects of reduction in amyloid levels on cognitive change by drug type (antibody versus small molecule drugs).

\section{Methods}

Data sources

On 9 May 2019, we searched the Alzheimer Research Forum (alzforum.org) for "Amyloid-Related" drugs for "Alzheimer's Disease" and "Mild Cognitive Impairment", which yielded a comprehensive list. We then searched for trials of these drugs on ClinicalTrials. gov and restricted our searches to trials for "Alzheimer's Disease" or "Mild Cognitive Impairment" that were "completed," "terminated," or "active, not recruiting." On the basis of information available on ClinicalTrials. gov, we excluded trials that did not have a placebo control and trials that did not quantify measures of brain amyloid using the standardized uptake value ratio obtained from amyloid positron emission tomography and change in a cognitive score within randomization arms. We added information on three trials that became available during the analysis and met the inclusion criteria.

\section{Study selection}

We excluded trials for which the evidence indicated no plausible differences in standardized uptake value ratio as a result of treatment (one trial of ACC-001 (vanutide cridificar); Janssen Biotech, Horsham, PA):
NCT01227564). When available, we obtained data from ClinicalTrials.gov, peer reviewed publications, or other publicly available materials, such as press releases. When data were unavailable online, we contacted pharmaceutical companies directly using telephone and email contact information posted on ClinicalTrials.gov. For the BAN2401 (lecanemab; Biogen, Cambridge, MA; Eisai, Tokyo, Japan) trial (NCT01767311), ${ }^{15}$ we obtained data from the study contact at Eisai pharmaceuticals. For the two trials of aducanumab (Biogen, Cambridge, MA), EMERGE (NCT02484547) and ENGAGE (NCT02477800), a press release was available in a portable document format with raw data (standard errors were measured from graphs). This analysis is based on all data available to us on 30 April 2020. We assumed that studies with no data available were missing completely at random. Results for a drug with notable cognitive benefits would almost certainly be publicly reported, so if the missing completely at random assumption was violated, any bias would likely be in a direction favorable to amyloid.

\section{Data extraction and synthesis}

Our approach combined instrumental variables analyses of each trial with a meta-analysis of effects across trials. This method allowed us to estimate the effect of amyloid reduction on cognitive change for each trial and to combine results for trials with different durations of follow-up. Analyses use maximum likelihood estimation. Supplementary appendices 1 and 3 provide additional details on our methods, and simulations showing unbiased results with valid confidence intervals.

First, we estimated effects within drug categories to assess evidence that the effects of reducing amyloid levels on cognitive change might differ based on the mechanism of amyloid reduction. A pooled estimate was then derived. To account for differences in populations enrolled in each trial and time in follow-up, we estimated separately for each trial how much change in cognition would be expected if the standardized uptake value ratio had not changed. In pooling results across trials, we assume that the effect on cognition of reducing amyloid levels does not vary with mechanism by which amyloid $\beta$ is targeted-that is, by drug. Furthermore, our analysis is premised on the assumption that these drugs primarily act through reducing amyloid levels. If a drug effectively both reduces amyloid levels and improves cognition through other mechanisms, our estimates would attribute this to efficacy of amyloid reduction in slowing cognitive decline.

We performed sensitivity analyses restricted to antibody drugs and with and without the unpublished trials of BAN2401 and aducanumab. Supplementary appendix 1 provides additional sensitivity analyses. The analysis was repeated using alternative cognitive outcomes (clinical dementia rating scalesum of boxes (CDR-SB) and various versions of the Alzheimer's disease assessment scale-cognitive subscale (ADAS-Cog)), when available. We also 
conducted sensitivity analyses stratified by the tracer used in amyloid positron emission tomography and applied a scaling transformation to deal with the possibility that estimates from different tracers were not comparable. ${ }^{16}$ Lastly, we considered the effect of a hypothetical additional large and successful trial on our pooled results. To provide a comparison for the clinical relevance of the effect sizes, we used a similar estimation procedure to estimate the effect of carrier status of the apolipoprotein E (APOE) $\& 4$ allele on annual change in cognition, based on the trials for which stratified cognitive outcomes were available.

Evidence from new trials, or updates of existing trials, are frequently released. The web based interface at https://amyloidintegratingevidence.shinyapps.io/ Shiny/ allows for a recalculation of results with either updated data or new data when available.

\section{Patient and public involvement}

This instrumental variable meta-analysis was done without patient involvement. Patients were not invited to comment on the study design and were not consulted to develop patient relevant outcomes or interpret the results. Patients were not invited to contribute to the writing or editing of this document for readability or accuracy. Two family members of people living with Alzheimer's disease provided valuable feedback on the framing and interpretation of results.

\section{Results}

Overall, 196 studies were identified that potentially met the inclusion criteria (fig 1). Two authors reviewed these trials, and 34 trials seemed likely to meet the inclusion criteria based on outcomes listed on ClinicalTrials.gov. Data from 14 (including 40 randomization arms) of these 34 trials were obtained, and data from the other 20 trials were requested from pharmaceutical companies by telephone or email but not obtained. Of the 20 eligible trials not included in this analysis, seven were classed as "completed," five as "terminated," and eight as "active, not recruiting." The 14 trials for which publicly available data were obtained tested the following drugs: bexarotene (Targretin; Ligand Pharmaceuticals, San Diego, CA; ReXceptor, Cleveland, $\mathrm{OH}$ ), solanezumab (three trials ${ }^{17}$; LY2062430; Eli Lilly, Indianapolis, IN), LY450139 (Semagacestat, two trials; Eli Lilly, Indianapolis, IN), gantenerumab (Chugai Pharmaceutical, Tokyo, Japan; Hoffmann-La Roche, Basel, Switzerland), bapineuzumab (two trials, three clinical trial numbers $^{12}{ }^{18-20}$; Janssen Biotech, Philadelphia, PA; Pfizer, New York, NY), Verubecestat (two trials; MK-8931, Merck Sharp \& Dohme, Kenilworth, NJ), BAN2401 (lecanemab; Biogen, Cambridge, MA; Eisai, Tokyo, Japan), ${ }^{131521}$ and aducanumab (Biogen, Cambridge, MA). ${ }^{22}$ All but two of these trials reported change in mini-mental state examination (MMSE) score as a cognitive outcome; one trial, of Verubecestat (NCT01953601), reported the clinical dementia rating scale-sum of boxes, and the trial of BAN2401 reported the Alzheimer's disease composite score (ADCOMS).
To provide a common scale, we converted changes in these cognitive measures to change in MMSE scores (see appendix 4 for details). ${ }^{23}$ When pooling trials of solanezumab, only two independent trials were used, because the third trial was an extension of an earlier trial. Appendix 2 summarizes the trials included in the aggregated analysis and where data were obtained.

The drugs found most effective at reducing amyloid did so by 0.2 to 0.3 standardized uptake value ratio units, whereas some drugs reduced amyloid levels by far less. The current study therefore presents estimated effects on cognition associated with a 0.1 unit reduction in standardized uptake value ratio. Figure 2 shows the estimated effect of a 0.1 reduction in standardized uptake value ratio on change in MMSE scores for eight drugs and 14 trials, with 95\% confidence intervals. Estimates from these trials were consistent with no effect of change in amyloid levels on MMSE score. When results from the 14 trials were pooled, the estimated effect of a 0.1 unit reduction in amyloid standardized uptake value ratio on the MMSE was 0.03 (95\% confidence interval -0.06 to 0.1 ) points. Pooling by drug type (antibody and small molecule) also yielded results consistent with no effect of amyloid reduction on cognition.

Individual trial results are also reported using various versions of the Alzheimer's disease assessment scale-cognitive subscale and individual and pooled results using the clinical dementia rating scale-sum of boxes (see appendix 1). Using these alternative cognitive measures, a single trial (EMERGE) showed a statistically significant effect of reduction in amyloid levels on cognitive change. For the clinical dementia rating scale-sum of boxes it was possible to pool across most trials ( $n=11)$, and the finding was not statistically significant. Sensitivity analyses stratified by tracer and applying transformations to tracer values indicated similarly null results (see appendix 1). As amyloid changed little in some trials (see appendix 1), the overall null findings of the current study could be sensitive to the addition of a highly successful trial. We evaluated how the pooled estimate would change with the addition of a new, hypothetical trial (similar to the EMERGE trial with respect to size, effect on amyloid, and standard errors). A hypothetical trial was defined that compared a low dose treatment group that decreased the standardized uptake value ratio by -0.15 and increased the MMSE score by 0.7 relative to the placebo group, and compared a high dose treatment group that decreased the standardized uptake value ratio by -0.30 and increased the MMSE score by 1.4 relative to the placebo group. The pooled estimate of the effect of a 0.1 reduction in standardized uptake value ratio on MMSE score incorporating this trial plus all previous results would be 0.094 (95\% confidence interval 0.0038 to 0.18 ).

To contextualize findings, effect estimates were compared with the effect of carrying an APOE- $\varepsilon 4$ allele on annual cognitive decline. The estimated effect on annual change in MMSE score of carrying an APOE- 84 allele from trials $2 \mathrm{a}$ and $2 \mathrm{~b}$ of bapineuzumab is -0.7 


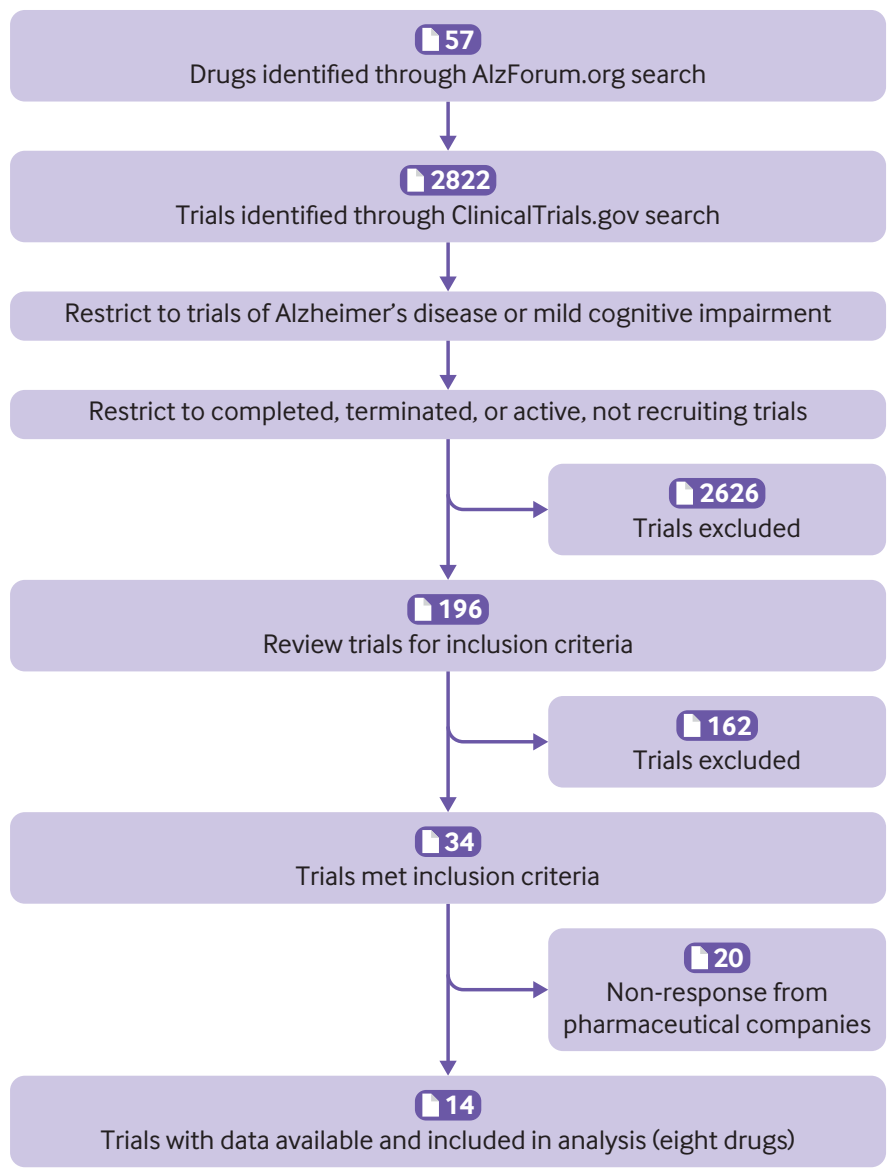

Fig 1 | Flowchart of trial exclusions and inclusions amyloid level on cognitive decline, but point estimates were variable and usually had wide confidence intervals. However, even the largest potential benefit consistent with the upper bound of the $95 \%$ confidence interval of our pooled estimate was small compared with the annual effect of APOE- $\varepsilon 4$ on decline in MMSE score.

\section{Strengths and limitations of this study}

Combining results from several studies is more precise than providing results from individual trials. The pooled estimate in our study indicates that reduction of amyloid levels within the time frame of the conducted trials is unlikely to provide substantial cognitive benefits. Among all trials, unpublished reports on aducanumab and BAN2401 indicated statistically significant benefit in at least one cognitive measure in intention-to-treat analyses. As noted in the external review in the FDA filing of aducanumab, ${ }^{25}$ these associations should be interpreted with caution because of the potential for a chance finding. Such chance findings become more likely as the number of trials targeting the same mechanism increases. It is therefore critical to consider pooled evidence from all available trials to contextualize results for any single trial.

Some caveats exist to this interpretation. First, we assumed that changes in cognition as a result of randomization to drug treatment were fully mediated by reductions in amyloid $\beta$. It is plausible, however, that reductions in amyloid $\beta$ improve cognitive outcomes but that the drugs evaluated negatively affected cognition through other mechanisms. We cannot rule out this possibility, but given the precision of our null effect estimate, the adverse direct effects would need to precisely counterbalance the positive effects of amyloid reduction, which is unlikely.

Second, we focused on the MMSE because it was reported by almost all the identified trials. Though MMSE has low sensitivity to cognitive deterioration in adults with normal cognition, nearly all these studies enrolled cognitively impaired participants who manifested deterioration in MMSE scores, indicating that the MMSE is sensitive to changes in cognition in the trial populations. In our sensitivity analyses of alternative cognitive outcomes (see appendix 1), evaluating only data from aducanumab for the Alzheimer's disease assessment scale-cognitive subscale and clinical dementia rating scale-sum of boxes cognitive outcomes, reduction in amyloid levels seemed to be effective at slowing cognitive decline. However, we also derived a pooled estimate for clinical dementia rating scale-sum of boxes for those trials reporting this outcome, and the pooled estimate was also null.

Third, after review of publicly available reports and contact with pharmaceutical companies, we were unable to obtain data for 20 trials that met our eligibility criteria. Most companies would presumably complete and post trial results if the drug showed promising findings, so it is unlikely that trials indicate that reducing amyloid levels with drug treatment has, at most, a small effect on cognition as measured with the MMSE. Findings from these trials individually were consistent with no effect of change in 


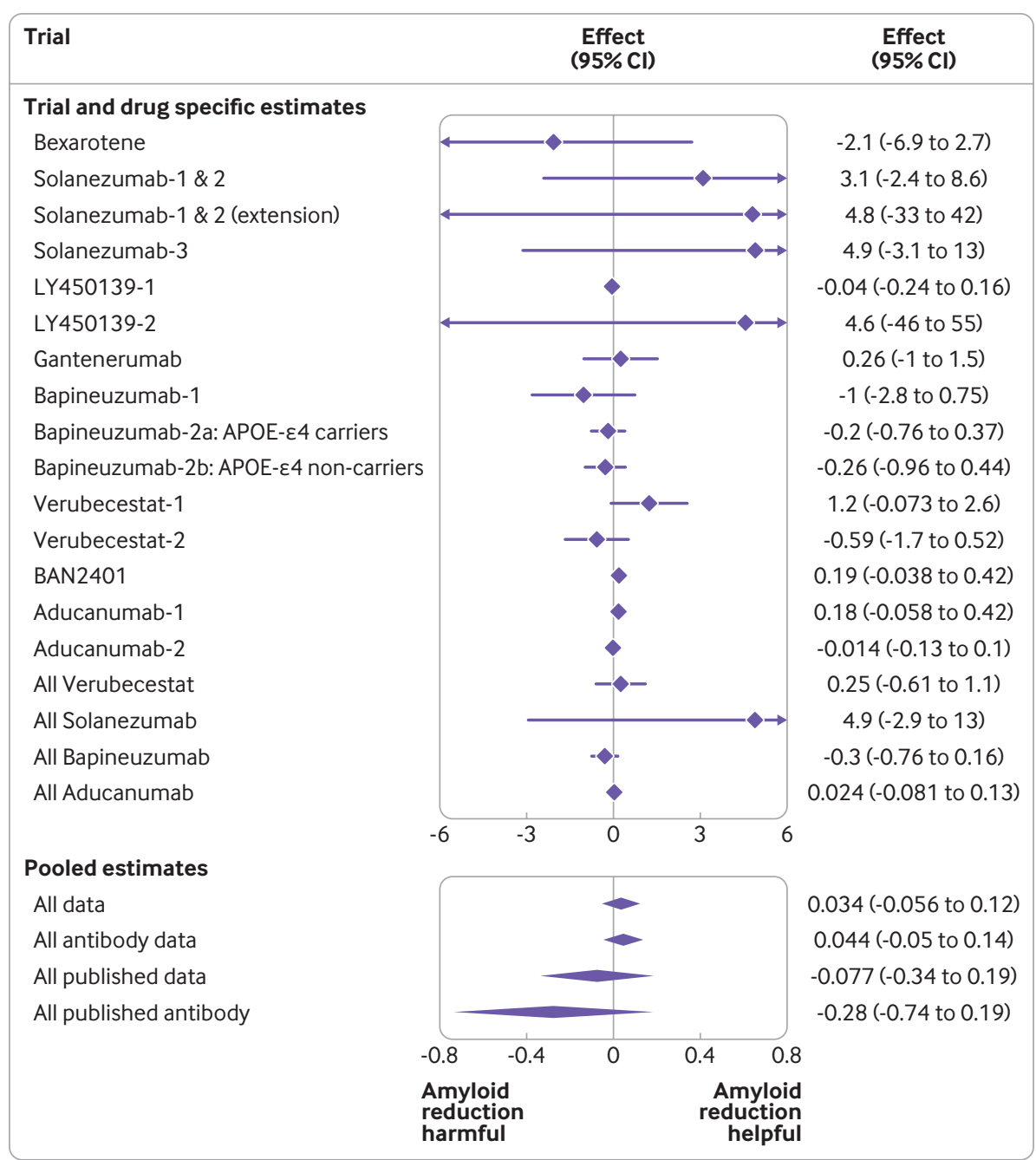

Fig 2 | Forest plot of estimated effects ( $95 \%$ confidence intervals) of a 0.1 decrease in standardized uptake value ratio on mini-mental state examination score for each trial and drug (top panel) and pooled across all drugs and by drug type (bottom panel). Trials of BAN2401 (lecanemab) and aducanumab are unpublished and were excluded from the "all published antibody" category. Centre and width of diamonds represent pooled estimates and $95 \%$ confidence intervals, respectively. The numbered key shows multiple trials of the same drug (see appendix table S2 for clinical trial numbers)

drugs evaluated in trials with no available results showed evidence of benefit of amyloid reduction. Any bias from omitting data for these studies is likely to be in favor of a beneficial effect of amyloid reduction. Our null results should thus be interpreted as an optimistic estimate for the cognitive benefit of amyloid reduction based on data from randomized controlled trials to date. Data for the trials classed as "active, not recruiting" might become available in the future.

Fourth, another limitation of our study is that we did not have access to full reports, so error in the input data is possible. To address this, we have published an interactive version of our analysis online at https:// amyloidintegratingevidence.shinyapps.io/Shiny/. The interface is autopopulated with the values we used in the analysis for each trial, and we provide the option of editing those values or including a hypothetical trial. Thus, input values can be manually modified and new data can be added to recalculate individual trial and pooled estimates under different assumptions.

Finally, we did not account for covariance between measured cognition and measured standardized uptake value ratio that might be induced if other factors influence amyloid levels and independently affect cognition because covariance was not reported. Simulation results indicate that this is unlikely to appreciably affect our estimates (see appendix 3).

\section{Implications}

Drugs that reduce brain amyloid levels comprise a large fraction of pharmaceuticals in the development pipeline for the prevention and treatment of Alzheimer's disease. Evidence for reduction in amyloid levels is routinely provided as evidence of target engagement for new drugs and support for the promise of efficacy in improving cognitive outcomes. Over the past two decades, numerous drugs that target amyloid have been evaluated in phase I, II, and III trials. Although 
two drugs seem promising in post hoc analyses, the data remain unpublished on ClinicalTrials.gov or in a peer reviewed journal. Null findings in trials of anti-amyloid drugs call into question the viability of amyloid as a target for the prevention and treatment of Alzheimer's disease. However, each of these trials was evaluated individually, with no research combining the evidence from all trials to evaluate whether amyloid reduction is likely to improve cognitive outcomes.

These findings suggest that reduction in amyloid levels is unlikely to have notable cognitive benefits within the time frame of typical trials. This does not conclusively invalidate the amyloid cascade hypothesis because amyloid reduction might have delayed effects on cognition that only manifest years later. If this is the case, trials on amyloid reduction would need to substantially extend the typical follow-up period to detect any benefit. Amyloid reduction strategies might be effective in other patient populations-for example, people with early stage Alzheimer's disease. The hypothesis that people with a lower baseline amyloid burden experience greater cognitive benefit from reduction in amyloid levels could be evaluated with additional data from extant trials.

\section{Conclusions}

Combined results from 14 randomized controlled trials provide evidence that reduction in amyloid levels alone is unlikely to substantially slow cognitive decline within the follow-up period of most typical trials. The results of pooled estimates suggest that use of anti-amyloid drugs is not a viable strategy for the prevention or treatment of Alzheimer's disease and that other potential targets may merit more attention.

Contributors: SFA, MCP, and MMG conceived the project. MMG supervised the work. SFA, MMG, ETT, and SCZ discussed and planned the analysis and contributed to the supplemental information. SFA collected the data from online sources and SCZ checked this work. SFA and WDB independently reviewed trials for meeting inclusion criteria. ALG contacted pharmaceutical companies by phone and email to request additional data. SFA performed the analysis. SFA, MCP, and MMG contributed to data visualization. SCZ reviewed all code and built the web application. AMB, JJM, ERM, TJF, and FE contributed to the interpretation of results and writing of the discussion section. All authors discussed the results and contributed to the writing and editing of the manuscript. SFA and MMG are the guarantors. The corresponding author attests that all listed authors meet authorship criteria and that no others meeting the criteria have been omitted.

Funding: This work was supported by National Institutes of Health (NIH) National Institute on Aging (NIA). SFA, SCZ, MCP, ERM, and MMG were supported by NIH NIA grant R01AG057869. EJTT was supported by NIH NIA grant R01AG065276. WDB was supported by NIH NIA grants T32AG049663 and K01AG062722. ERM was supported by NIH NIA grant 5R00AG053410. The funders had no role in considering the study design or in the collection, analysis, interpretation of data, writing of the report, or decision to submit the article for publication.

Competing interests: All authors have completed the ICMJE uniform disclosure form at www.icmje.org/coi_disclosure.pdf and declare: support from the National Institutes of Health National Institute on Aging for the submitted work; no financial relationships with any organizations that might have an interest in the submitted work in the previous three years; no other relationships or activities that could appear to have influenced the submitted work.

Ethical approval: No individual level data are included in this manuscript. All data are aggregated data from clinical trials that are publicly available, with the exception of aggregated data for the trial of BAN2401 obtained from Eisai Pharmaceuticals.
Data sharing: Statistical code and dataset available from the first author at sarah.ackley@ucsf.edu.

The lead author (the manuscript's guarantor) affirms that the manuscript is an honest, accurate, and transparent account of the study being reported; that no important aspects of the study have been omitted; and that any discrepancies from the study as planned (and, if relevant, registered) have been explained.

Dissemination to participants and related patient and public communities: Results will be disseminated using social media (Twitter), at conferences (Alzheimer's Association International Conference, Methods in Longitudinal Dementia Research, and Society for Epidemiologic Research), and to stakeholders at non-profit organizations.

Provenance and peer review: Not commissioned; externally peer reviewed.

This is an Open Access article distributed in accordance with the Creative Commons Attribution Non Commercial (CC BY-NC 4.0) license, which permits others to distribute, remix, adapt, build upon this work non-commercially, and license their derivative works on different terms, provided the original work is properly cited and the use is noncommercial. See: http://creativecommons.org/licenses/by-nc/4.0/.

1 Hardy JA, Higgins GA. Alzheimer's disease: the amyloid cascade hypothesis. Science 1992;256:184-5. doi:10.1126/ science. 1566067

2 Hardy J. Alzheimer's disease: the amyloid cascade hypothesis: an update and reappraisal. / Alzheimers Dis 2006:9(Suppl):151-3.

Selkoe DJ, Hardy J. The amyloid hypothesis of Alzheimer's disease at 25 years. EMBO Mol Med 2016;8:595-608. doi:10.15252/ emmm.201606210

4 Karran E, Mercken M, De Strooper B. The amyloid cascade hypothesis for Alzheimer's disease: an appraisal for the development of therapeutics. Nat Rev Drug Discov 2011;10:698-712. doi:10.1038/ nrd3505

5 Näslund J, Haroutunian V, Mohs R, et al. Correlation between elevated levels of amyloid $\beta$-peptide in the brain and cognitive decline. IAMA 2000:283:1571-7. doi:10.1001/jama.283.12.1571

6 Forsberg A, Engler H, Almkvist O, et al. PET imaging of amyloid deposition in patients with mild cognitive impairment. Neurobiol Aging 2008;29:1456-65. doi:10.1016/j. neurobiolaging.2007.03.029

7 Nelson PT, Alafuzoff I, Bigio EH, et al. Correlation of Alzheimer disease neuropathologic changes with cognitive status: a review of the literature. J Neuropathol Exp Neurol 2012;71:362-81. doi:10.1097| NEN.Ob013e31825018f7

8 Cummings J, Ritter A, Zhong K. Clinical trials for disease-modifying therapies in Alzheimer's disease: A primer, lessons learned, and a blueprint for the future. J Alzheimers Dis 2018;64(s1):S3-22. doi:10.3233/JAD-179901

9 Selkoe DJ. Resolving controversies on the path to Alzheimer's therapeutics. Nat Med 2011;17:1060-5. doi:10.1038/nm.2460

10 Mullane K, Williams M. Alzheimer's therapeutics: continued clinical failures question the validity of the amyloid hypothesis-but what lies beyond?Biochem Pharmacol 2013;85:289-305. doi:10.1016/i. bcp.2012.11.014

11 Clarke JR, Ribeiro FC, Frozza RL, De Felice FG, Lourenco MV. Metabolic dysfunction in Alzheimer's disease: from basic neurobiology to clinical approaches. J Alzheimers Dis 2018;64(s1):S405-26. doi:10.3233/JAD-179911

12 Abushouk Al, Elmaraezy A, Aglan A, et al. Bapineuzumab for mild to moderate Alzheimer's disease: a meta-analysis of randomized controlled trials. BMC Neurol 2017;17:66. doi:10.1186/s12883017-0850-1

13 Lannfelt L, Möller C, Basun $\mathrm{H}$, et al. Perspectives on future Alzheimer therapies: amyloid- $\beta$ protofibrils - a new target for immunotherapy with BAN2401 in Alzheimer's disease. Alzheimers Res Ther 2014:6:16. doi:10.1186/alzrt246

14 Baiocchi M, Cheng J, Small DS. Instrumental variable methods for causal inference. Stat Med 2014;33:2297-340. doi:10.1002/ $\operatorname{sim} .6128$

15 Satlin A, Wang J, Logovinsky V, et al. Design of a Bayesian adaptive phase 2 proof-of-concept trial for BAN2401, a putative diseasemodifying monoclonal antibody for the treatment of Alzheimer's disease. Alzheimers Dement (N Y) 2016;2:1-12. doi:10.1016/i. trci.2016.01.001

16 Bourgeat P, Doré V, Fripp J, et al, AIBL research group. Implementing the centiloid transformation for ${ }^{11} \mathrm{C}$-PiB and $\beta$-amyloid ${ }^{18} \mathrm{~F}$-PET tracers using CapAIBL. Neuroimage 2018;183:387-93. doi:10.1016/j. neuroimage.2018.08.044

17 Imbimbo BP, Ottonello S, Frisardi V, et al. Solanezumab for the treatment of mild-to-moderate Alzheimer's disease. Expert Rev Clin Immunol 2012;8:135-49. doi:10.1586/eci.11.93 
18 Salloway S, Sperling R, Fox NC, et al, Bapineuzumab 301 and 302 Clinical Trial Investigators. Two phase 3 trials of bapineuzumab in mild-to-moderate Alzheimer's disease. N Engl J Med 2014;370:322 33. doi:10.1056/NEJMoa1304839

19 Salloway S, Sperling R, Gilman S, et al, Bapineuzumab 201 Clinical Trial Investigators. A phase 2 multiple ascending dose trial of bapineuzumab in mild to moderate Alzheimer disease. Neurology 2009;73:2061-70. doi:10.1212/WNL.0b013e3181c67808

20 Rinne JO, Brooks DJ, Rossor MN, et al. 11C-PiB PET assessment of change in fibrillar amyloid- $\beta$ load in patients with Alzheimer's disease treated with bapineuzumab: a phase 2, double-blind, placebocontrolled, ascending-dose study. Lancet Neurol 2010;9:363-72. doi:10.1016/S1474-4422(10)70043-0

21 Logovinsky V, Satlin A, Lai R, et al. Safety and tolerability of BAN2401--a clinical study in Alzheimer's disease with a protofibril selective $A \beta$ antibody. Alzheimers Res Ther 2016;8:14. doi:10.1186/ s13195-016-0181-2
22 Sevigny J, Chiao $P$, Bussière T, et al. The antibody aducanumab reduces A $\beta$ plaques in Alzheimer's disease. Nature 2016;537:50-6. doi:10.1038/nature19323

23 Balsis S, Benge JF, Lowe DA, Geraci L, Doody RS. How do scores on the ADAS-Cog, MMSE, and CDR-SOB correspond?Clin Neuropsychol 2015;29:1002-9. doi:10.1080/13854046.2015.11 19312

24 Howard J, Gumbrecht J. Drugmaker to seek approval for alzheimer's treatment. CNN. CableNews Network; 2019. https:// www.cnn.com/2019/10/22/health/biogen-alzheimers-drug-fda/ index.html.

25 FDA. Peripheral and central nervous system drugs advisory committee meeting. [cited 5 Dec 2020]. https://www.fda.gov/ media/143502/download

Supplementary information: appendices 